\title{
Enhancement of wireless positioning in outdoor suburban NLOS environment using hybrid- network-GPS systems
}

\author{
Saleh O Al-Jazzar
}

\begin{abstract}
This article will introduce a method for locating mobile stations (MSs) in outdoor suburban non-line-of-sight (NLOS) environment. The measurements used to locate the MS are taken from three base stations and a satellite. Such a setup of measurements is named the hybrid-network-GPS system. The proposed method uses constraint nonlinear optimization to minimize the NLOS error. The problem is simplified to three independent nonlinear equations of three unknowns, then it is solved to find the MS location. Numerical simulations are introduced to assess the performance of the proposed method compared with other positioning algorithms.
\end{abstract}

Keywords: NLOS, GPS, TOA, constrained minimization

\section{Introduction}

The well-known non-line-of-sight (NLOS) problem in wireless location has gained a great attention in the last decade. An important aspect of this problem is the huge error it induces in locating wireless devices. Thus, it is of interest to develop wireless positioning algorithms that will minimize the NLOS error.

In the literature, many articles addressed the NLOS problem. These articles differed in their approaches to solve the problem. The articles in [1-6] used nonstatistical methods to locate the mobile station (MS) using network based measurements. In [1], the authors propose using linear-programming method to locate the MS in NLOS environments. The authors in [2], linearize the inequality of range models corrupted with NLOS errors for wireless positioning. In [3], the authors propose a constrained-optimization algorithm to locate the MS using the sequential quadratic programming algorithm. The authors in [4] propose a geometry-assisted location estimation algorithm utilizing the different geometric layouts between the MS and the base stations (BSs). In [5], the authors propose a constrained optimization technique to locate the MS. The method in [6] is a constrained nonlinear optimization approach, with constraints derived from the

Correspondence: s.jazzar@uoh.edu.sa

University of Ha'il, Ha'il, Saudi Arbia geometry of the cell layout and range measurements. Satellite assisted techniques were also proposed in $[7,8]$ for wireless positioning but they did not assume NLOS environment and measurements were taken at more than one satellite.

This article uses the hybrid-network-GPS system to minimize the NLOS error when locating the MS. The proposed method is named the hybrid-network-GPS constrained algorithm (HNGPS-CA) which depends on minimizing a constraint objective function to locate the MS. The proposed objective function used in the HNGPS-CA method has the advantage of guaranteed convexity. This advantage is not guaranteed in the regularly used least square (LS) objective function which might be non-convex in some cases as will be clarified in Section 4. The environment considered in this article is the outdoor suburban NLOS environment. We will consider that there are three time of arrival (TOA) measurements available from three BSs and only one TOA measurement from the satellite.

The rest of the article is organized as follows: Section 2 presents the problem formulation. Section 3 presents the HNGPS-CA algorithm. In Section 4, some insight on the convexity of the objective function will be provided. Section 5 shows simulation results for the proposed method. Conclusions are presented in Section 6. 


\section{Problem formulation}

Assume that there is a transmitting MS (location $\psi_{m}=$ $\left.\left(x_{\mathrm{ms}}, y_{\mathrm{ms}}, 0\right)\right)$ with the TOA measurements taken at the receiving $\mathrm{BS}_{i}$ (location $\psi_{i}=\left(x_{i}, y_{i}, 0\right)$, where $i=1,2,3$ which is an index for the BS) and at one GPS satellite (location $\left.\psi_{s}=\left(x_{s}, y_{s}, z_{s}\right)\right)$. Then, the true distance between the MS and $\mathrm{BS}_{i}$ is

$$
R_{i}=\left\|\psi_{m}-\psi_{i}\right\|=\sqrt{\left(x_{\mathrm{ms}}-x_{i}\right)^{2}+\left(y_{\mathrm{ms}}-y_{i}\right)^{2}} \text { for } i=1,2,3
$$

The true distance between the MS and the GPS satellite is

$$
R_{s}=\left\|\psi_{m}-\psi_{s}\right\|=\sqrt{\left(x_{\mathrm{ms}}-x_{s}\right)^{2}+\left(\gamma_{\mathrm{ms}}-\gamma_{s}\right)^{2}+\left(0-z_{s}\right)^{2}} .
$$

A layout of the MS, multiple BSs and satellite geometry is shown in Figure 1.

Since the consideration for this article is the outdoor suburban environment, then it is reasonable to assume that it is highly unlikely that there will be obstacles between the MS and the GPS satellite in such an environment. Thus, the measured distance between the MS and the GPS satellite $\left(\tilde{R}_{s}\right)$ will be assumed to be the same as the true distance $\left(R_{s}\right)$ added to it the measurement noise $\left(\mu_{s}\right)$, i.e., $\tilde{R}_{s}=R_{s}+\mu_{s}$. The measurement

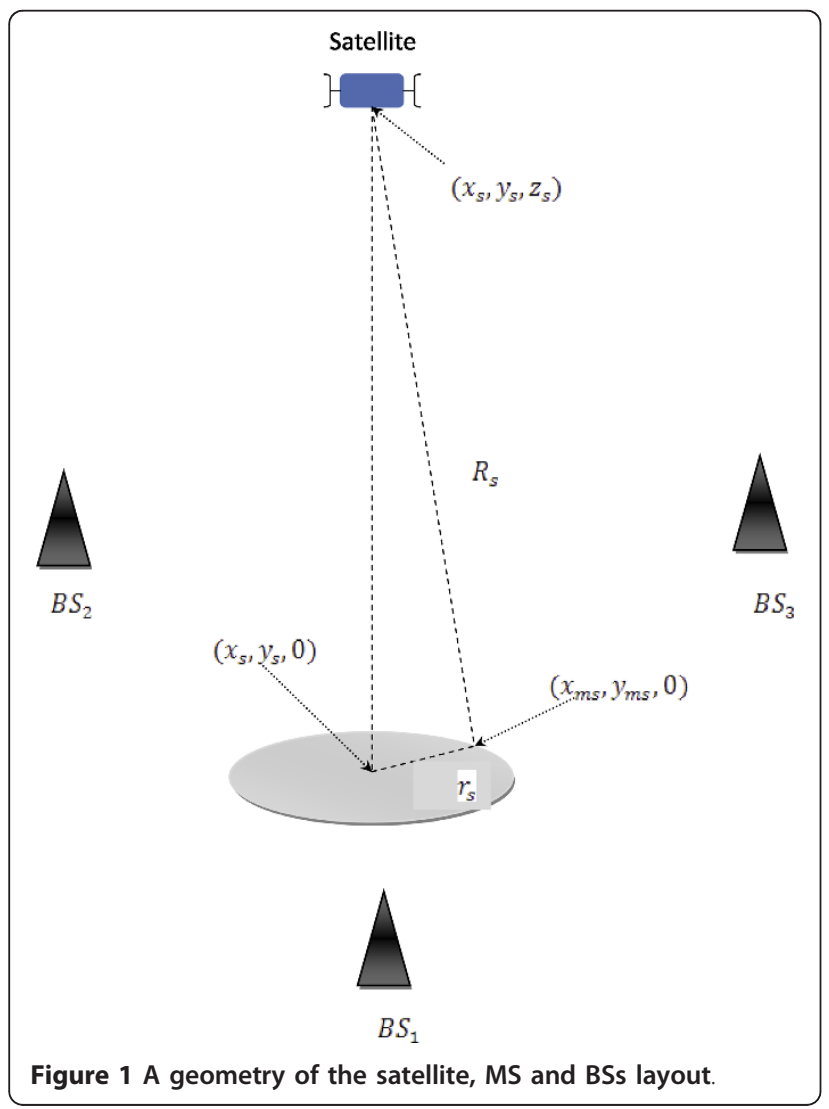

noise $\left(\mu_{s}\right)$ is assumed to be additive white Gaussian noise (AWGN) of zero mean and variance of $\sigma_{s}^{2}$.

This assumption is not valid for the connection between the MS and the BSs where it is very likely that obstacles exist in the MS-BSs connections. Thus, we will consider that the distance measurements at the BSs will have NLOS error (unlike the measurement made at the MS-GPS connection).

The measured distance at $\mathrm{BS}_{i}$ (between $\mathrm{BS}_{i}$ and MS) would be

$$
l_{i}=R_{i}+\eta_{i}+\mu_{i}
$$

where $\eta_{i}$ is the effective NLOS component and $\mu_{i}$ is the measurement noise, which can be considered as AWGN of zero mean and variance of $\sigma_{i}^{2}$. Since the NLOS causes the signal to arrive from a path which is longer than the true distance, then $\eta_{i} \geq 0$. It is generally considered that the NLOS error $\left(\eta_{i}\right)$ is usually much more severe than the measurement noise $\left(\mu_{i}\right)$.

Another distance measurement is obtained by calculating the time the wave takes to go from the GPS satellite-to-MS-to-BS $\mathrm{i}_{\mathrm{i}}$ which we will call $\left(\bar{l}_{i}\right)$, as follows:

$$
\overline{l_{i}}=l_{i}+\tilde{R}_{s} .
$$

This distance can be practically obtained by assuming the MS will receive the signal from the satellite and then transmits the TOA measurement to $\mathrm{BS}_{i}$ or retransmits the same signal and the $\mathrm{BS}_{i}$ computes the total distance $\left(\bar{l}_{i}\right)$.

Since the GPS satellite is located far from the earth surface (i.e., $R_{s}$ is a huge distance when compared to cells dimensions), then $\left(\bar{l}_{i}\right)$ is much greater than $l_{i}$ (i.e., $\bar{l}_{i}>>l_{i}$ ).

Next, the HNGPS-CA method is presented to locate the MS accurately in the presence of the NLOS error.

\section{Hybrid-network-GPS constrained algorithm}

The HNGPS-CA method is based on minimizing a constraint objective function to estimate the MS location. The objective function is defined as the squared summation of the differences between $\left(\bar{l}_{i}\right)$ and $R_{i}$ (for $i=1,2$, 3 ). In other words, the objective function that will be minimized is

$$
F_{\mathrm{HNGPS}-\mathrm{CA}}\left(x_{\mathrm{ms}}, y_{\mathrm{ms}}\right)=\sum_{i=1}^{3} \alpha_{i}\left(\overline{l_{i}}-R_{i}\right)^{2}
$$

where $\alpha_{i}$ (for $i=1 ; 2$; $)$ are positive weighting factors for the objective function (i.e., $\alpha_{i}>0$ ). Each positive weighting factor $\left(\alpha_{i}\right)$ can be chosen separately, depending on the measurement accuracy [5] or the 
measurement geometry [6]. Recall that $R_{i}$ is function of $x_{\mathrm{ms}}$ and $y_{\mathrm{ms}}$ as shown in (1), and they are the variables over which $F_{\text {HNGPS-CA }}\left(x_{\mathrm{ms}}, y_{\mathrm{ms}}\right)$ in (5) is minimized.

The reason for choosing such an objective function rather than the regular LS objective function given by

$$
F_{\mathrm{LS}}\left(x_{\mathrm{ms}}, y_{\mathrm{ms}}\right)=\sum_{i=1}^{3} \alpha_{i}\left(l_{i}-R_{i}\right)^{2}
$$

is the fact that using the objective function in (5) is assured to be convex. Whereas, the one in (6) might be non-convex in some cases as will be explained in Section 4.

Now, let us consider the distance $R_{s}$ which represents the distance between the MS and the GPS satellite (which is very close to its measured value $\tilde{R}_{S}$ if we neglect the measured noise $\mu_{s}$ remembering that $R_{s}>\mu_{s}$ ). The locus of $R_{s}$ will be a circle on the earth plane (the $x-y$ plane) as shown in Figure 1. This circle is centered at $\left(x_{s}, y_{s}\right)$ with radius $r_{s}=\sqrt{\left(R_{s}^{2}-z_{s}^{2}\right)}$. Thus, the MS estimated coordinates must be located on this circle, i.e.,

$$
\left(x_{\mathrm{ms}}-x_{s}\right)^{2}+\left(y_{\mathrm{ms}}-y_{s}\right)^{2}=r_{s}^{2} .
$$

Thus, (7) provides an equality constraint on finding $\left(x_{\mathrm{ms}}, y_{\mathrm{ms}}\right)$ when applying the HNGPS-CA.

As mentioned earlier, we intend to estimate the MS location by minimizing the objective function in (5) taking into consideration the equality constraint in (7). This constraint minimization can be done using the Lagrange multipliers as follows:

$$
\zeta\left(x_{\mathrm{ms}}, \gamma_{\mathrm{ms}}, \lambda\right)=\sum_{i=1}^{3} \alpha_{i}\left(\bar{T}_{i}-R_{i}\right)^{2}+\lambda\left(\left(x_{\mathrm{ms}}-x_{s}\right)^{2}+\left(y_{\mathrm{ms}}-y_{s}\right)^{2}-r_{s}^{2}\right)
$$

where $\lambda$ is the Lagrange multiplier.

To find the minimum of $\zeta\left(x_{\mathrm{ms}}, y_{\mathrm{ms}}, \lambda\right)$, we take its derivative with respect to the variables upon which $\zeta\left(x_{\mathrm{ms}}, y_{\mathrm{ms}}, \lambda\right)$ depends, i.e., $\left(x_{\mathrm{ms}}, y_{\mathrm{ms}}, \lambda\right)$ separately and equate each result to zero,

$$
\begin{gathered}
\frac{\partial \zeta\left(x_{\mathrm{ms}}, y_{\mathrm{ms}}, \lambda\right)}{\partial x_{\mathrm{ms}}}=-\sum_{i=1}^{3} \alpha_{i} \frac{\left(\bar{l}_{i}-R_{i}\right)\left(x_{\mathrm{ms}}-x_{s}\right)}{R_{i}}+\lambda\left(x_{\mathrm{ms}}-x_{s}\right)=0, \\
\frac{\partial \zeta\left(x_{\mathrm{ms}}, y_{\mathrm{ms}}, \lambda\right)}{\partial \gamma_{\mathrm{ms}}}=-\sum_{i=1}^{3} \alpha_{i} \frac{\left(\bar{l}_{i}-R_{i}\right)\left(y_{\mathrm{ms}}-\gamma_{s}\right)}{R_{i}}+\lambda\left(y_{\mathrm{ms}}-\gamma_{s}\right)=0
\end{gathered}
$$

and

$$
\frac{\partial \zeta\left(x_{\mathrm{ms},} y_{\mathrm{ms},} \lambda\right)}{\partial \lambda}=\left(x_{\mathrm{ms}}-x_{s}\right)^{2}+\left(y_{\mathrm{ms}}-y_{s}\right)^{2}-r_{s}^{2}=0 .
$$

Thus, (9), (10), and (11) provide three independent nonlinear equations with three unknowns $\left(x_{\mathrm{ms}}, y_{\mathrm{ms}}, \lambda\right)$. These equations can be solved to find the unknowns especially the MS location coordinates $\left(x_{\mathrm{ms}}, y_{\mathrm{ms}}\right)$. We will use numerical techniques to solve these equations. Here, we use fsolve.m in MATLAB to solve the set of nonlinear equations in (9), (10), and (11).

\section{Convexity of the objective functions}

As shown in Section 3, we use the objective function $F_{\text {HNGPS-CA }}\left(x_{\mathrm{ms}}, y_{\mathrm{ms}}\right)$ in (5) rather than the LS obcetive function $\left(F_{\mathrm{LS}}\left(x_{\mathrm{ms}}, y_{\mathrm{ms}}\right)\right)$ shown in (6). To verify this choice, let us look at the objective functions $F_{\text {HNGPS-CA }}$ $\left(x_{\mathrm{ms}}, y_{\mathrm{ms}}\right)$ and $F_{\mathrm{LS}}\left(x_{\mathrm{ms}}, y_{\mathrm{ms}}\right)$.

For sake of simplicity, from now on we will drop the argument $\left(x_{\mathrm{ms}}, y_{\mathrm{ms}}\right)$ in $F_{\mathrm{LS}}\left(x_{\mathrm{ms}}, y_{\mathrm{ms}}\right)$ and $F_{\mathrm{HNGPS}-\mathrm{CA}}\left(x_{\mathrm{ms}}\right.$, $\left.y_{\mathrm{ms}}\right)$. Thus, we can write (6) as follows

$$
F_{\mathrm{LS}}=\sum_{i=1}^{3} \alpha_{i} f_{i}
$$

where

$$
f_{i}=\left(l_{i}-R_{i}\right)^{2} .
$$

From the definition of $l_{i}$ in (3), the function $f_{i}$ can be simplified to

$$
f_{i}=\left(\eta_{i}+\mu_{i}\right)^{2} .
$$

Let us recall that we intend to minimize $\eta_{i}$ which always has a nonnegative value, i.e., $\eta_{i} \geq 0$. Also, $\eta_{i}$ is actually a distance and function of the estimated MS coordinates $\left(\hat{x}_{\mathrm{ms}}, \hat{y}_{\mathrm{ms}}\right)$, where $\{\wedge\}$ represents the estimated value of the variable, i.e., for example $\hat{v}$ is the estimated value of $v$. Since $\eta_{i}$ is a positive error which increases as the estimated MS coordinates $\left(\hat{x}_{\mathrm{ms}}, \hat{y}_{\mathrm{ms}}\right)$ get further from the actual MS coordinates $\left(x_{\mathrm{ms}}, y_{\mathrm{ms}}\right)$, then $\eta_{i}$ is a convex surface which has a minima (zero value) at $\left(x_{\mathrm{ms}}, y_{\mathrm{ms}}\right)$. Now, because $\mu_{i}$ is a noise signal it can have both negative and positive values. If it has a positive value then the surface $\eta_{i}+\mu_{i}$ has a value which is greater than zero, i.e., $\eta_{i}+\mu_{i}>0$ and has a minima at $\left(x_{\mathrm{ms}}, y_{\mathrm{ms}}\right)$. Thus, $f_{i}=\left(\eta_{i}+\mu_{i}\right)^{2}$ will be convex in this case and $F_{\mathrm{LS}}$ which is the summation of positively weighted convex functions $\left(f_{i}\right)$ for $i=1,2,3$ will be convex in this case as well [9]. Whereas, if $\mu_{i}$ is negative, i.e., $\mu_{i}<0$, then parts of the surface $\eta_{i}+\mu_{i}$ will be below zero and other parts will be above zero with the minimum located at $\left(x_{\mathrm{ms}}\right.$, $\left.y_{\mathrm{ms}}\right)$ to be below zero. When taking the square of $\eta_{i}+\mu_{i}$, i.e., $\left(\eta_{i}+\mu_{i}\right)^{2}$, then, the point $\left(x_{\mathrm{ms}}, y_{\mathrm{ms}}\right)$ will have a local maxima and the surface surrounding it will be decreasing until it hits the zero level. Then, the surface $\left(\eta_{i}+\mu_{i}\right)^{2}$ is reflected back to the increasing mode as we get further from the point $\left(x_{\mathrm{ms}}, y_{\mathrm{ms}}\right)$, causing $\left(\eta_{i}+\mu_{i}\right)^{2}$ to be a nonconvex surface, i.e., $f_{i}$ will be non-convex in this case, causing $F_{\mathrm{LS}}$ which is the summation of positively 
weighted non-convex functions $\left(f_{i}\right)$ for $i=1,2,3$ will be non-convex in this case [9]. This is illustrated in Figure 2. Thus, when minimizing $F_{\mathrm{LS}}$ we will reach an unwanted local minimum which is not located at $\left(x_{\mathrm{ms}}, y_{\mathrm{ms}}\right)$. So, there will be an error in locating the MS.

Similarly, $F_{\text {HNGPS-CA }}$ in (5) can be written as follows

$$
F_{\mathrm{HNGPS}-\mathrm{CA}}=\sum_{i=1}^{3} \alpha_{i} g_{i}
$$

where

$$
g_{i}=\left(\overline{l_{i}}-R_{i}\right)^{2} .
$$

From the definition of $\left(\bar{l}_{i}\right)$ in (4), the function $g_{i}$ can be simplified to

$$
g_{i}=\left(\eta_{i}+\mu_{i}+R_{s}+\mu_{s}\right)^{2} .
$$

Let us introduce the variable $h_{i}$ which is defined as

$$
h_{i}=\mu_{i}+R_{s}+\mu_{s} .
$$

Thus,

$$
g_{i}=\left(\eta_{i}+h_{i}\right)^{2} .
$$

Because $R_{s}$ is the distance between the MS and the satellite which is much greater than zero, i.e., $R_{s}>>0$, and because $R_{s} \gg>\mu_{i}$ and $R_{s}>>\mu_{s}$, then $h_{i}>>0$ for all realistic values of $\mu_{i}$ and $\mu_{s}$. Thus, the function $g_{i}$ is the square of the summation of a positive surface $\left(\eta_{i}\right)$ which has a minimum value of zero at $\left(x_{\mathrm{ms}}, y_{\mathrm{ms}}\right)$ and a positive variable $\left(h_{i}\right)$. So, $g_{i}$ is always a convex function and has a minimum value at $\left(x_{\mathrm{ms}}, y_{\mathrm{ms}}\right)$. This is illustrated in Figure 3 . Thus, $F_{\text {HNGPS-CA }}$ is convex as well because the positively weighted sum of convex functions is also convex

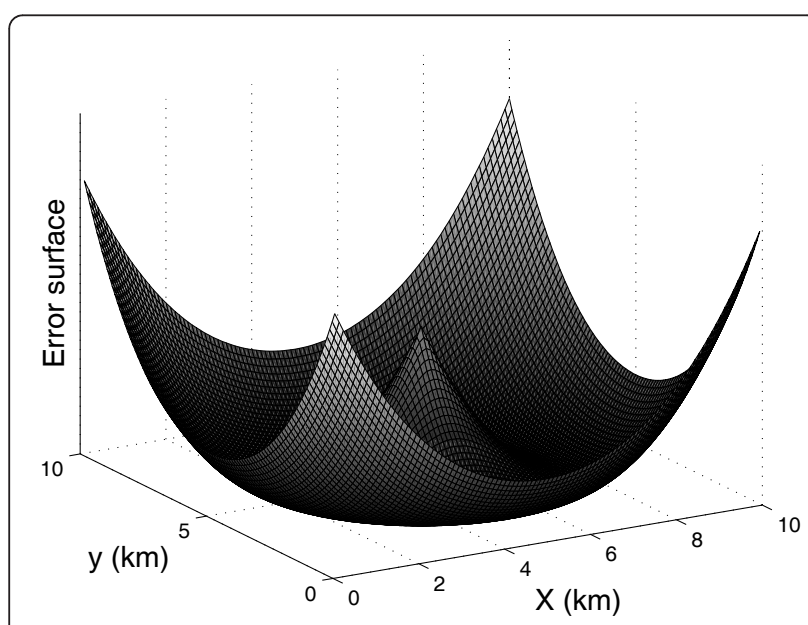

Figure 2 The error surface for $f_{i}$ when $\eta_{i}+\mu_{i}<0$ for some location region.

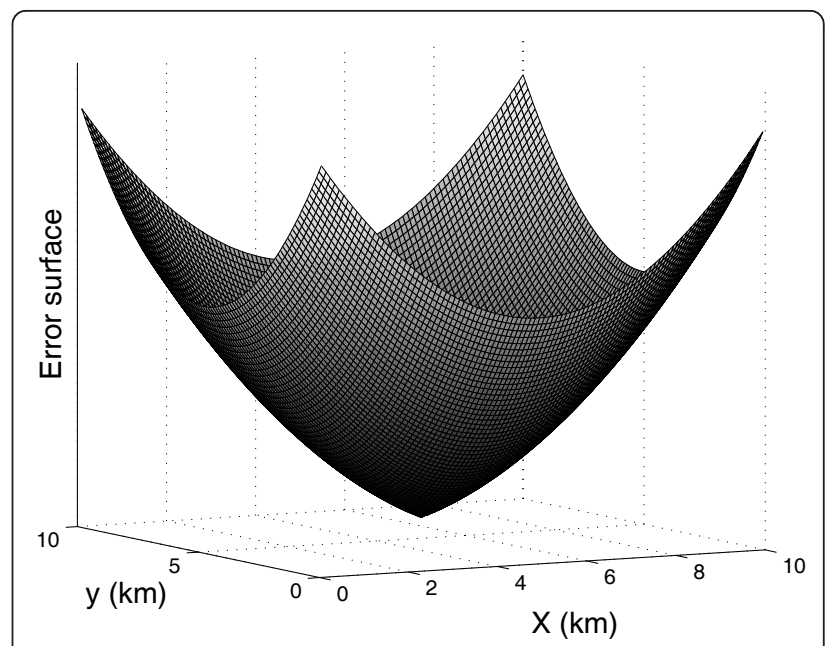

Figure 3 The error surface for $g_{i}$.

[9] (recall that $F_{\text {HNGPS-CA }}=\Sigma_{i=1}^{3} \alpha_{i} g_{i}$ ). This will assure that when minimizing $F_{\mathrm{HNGPS}-\mathrm{CA}}$ we will not reach an unwanted local minimum as the case for minimizing $F_{\mathrm{LS}}$, but rather we will reach the true MS location $\left(x_{\mathrm{ms}}, y_{\mathrm{ms}}\right)$.

\section{Simulation results}

The results in this section are averaged over 2,000 ensemble runs. The BSs locations are $(0,0),(8.66,0)$ and $(4.33,7.5)$ with all units in $\mathrm{km}$. The MS location is $\left(x_{\mathrm{ms}}, y_{m \mathrm{~s}}\right)$ where $x_{\mathrm{ms}}=4.33 \cdot(u+0.5)$ in $\mathrm{km}, y_{\mathrm{ms}}=0.5$. $(7.5+u)$ in $\mathrm{km}$ and $u$ is a random variable uniformly distributed in the region $[0,1]$. Two NLOS environment models are assumed. The first case considered to model the NLOS effect is the disk of scatterers (DOS) environments presented in [10]. In the DOS model, the scatterers are located on a solid circular disk of fixed radius $R_{d}$ with the MS at the center. The distance to a scatterer from the MS, $r_{\mathrm{DOS}}$, is uniformly distributed over $\left[0, R_{d}\right]$, and the angle is uniformly distributed over $[0,2 \pi]$. The radius $R_{d}$ is set to $300 \mathrm{~m}$. In the second case we assume that the NLOS error is a ratio of the measured distance. The reasoning behind this assumption is the fact that as the wave travels through a larger distance there will be a higher probability that the NLOS error will be larger. Thus, in this case $\eta_{i}=\beta l_{i}$ where $\beta$ is the NLOS error factor and it is assumed to be 0.3 in the simulations. The range measurement error is assumed to have $50 \mathrm{~m}^{2}$ variance, i.e, $\sigma_{s}^{2}=50 \mathrm{~m}^{2}$. The weight factors $\left(\alpha_{1}, \alpha_{2}, \alpha_{3}\right)$ in (9) and (10) are all set to 1.

The HNGPS-CA algorithm is compared with the range scaling algorithm (RSA) in [6] and the linear line of positions (LLOP) algorithm in [11]. Figures 4 and 5 show the error cumulative distribution function (CDF) of the different algorithms for the first and second NLOS environment cases, respectively. To show what 


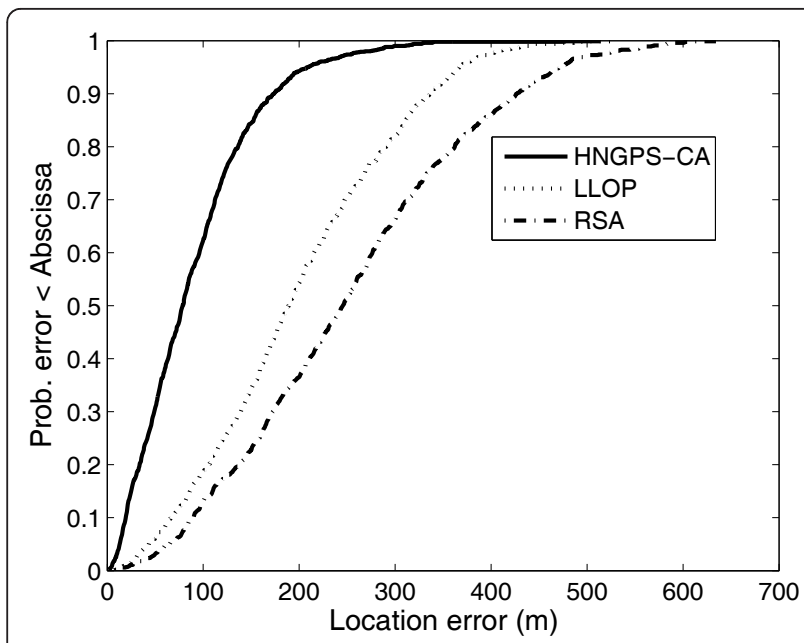

Figure 4 Comparisons of the CDF of the estimation error using the HNGPS-CA, the LLOP and the RSA algorithms in the first case NLOS environment model.

these CDFs mean in both figures, let us consider Figure 4 for example. The CDF curve in Figure 4 for the HNGPS-CA at the $100 \mathrm{~m}$ location error at the $x$-axis gives 0.59 at the $y$-axis which means that $59 \%$ of the time the error is below $100 \mathrm{~m}$, whereas, it was 18 and $12 \%$ for the RSA and LLOP algorithms, respectively, for the same location error $(100 \mathrm{~m})$. As for the location error of $200 \mathrm{~m}$, the HNGPS-CA, RSA and LLOP algorithms had an error of less than $200 \mathrm{~m}$ with a probability of 95,50 and $34 \%$, respectively. Thus, clearly the HNGPS-CA is giving better performance than the RSA and LLOP algorithms. The same conclusion can be achieved by looking at Figure 5. Thus, both figures indicate that the HNGPS-CA algorithm performs considerably better than both the LLOP and RSA algorithms.

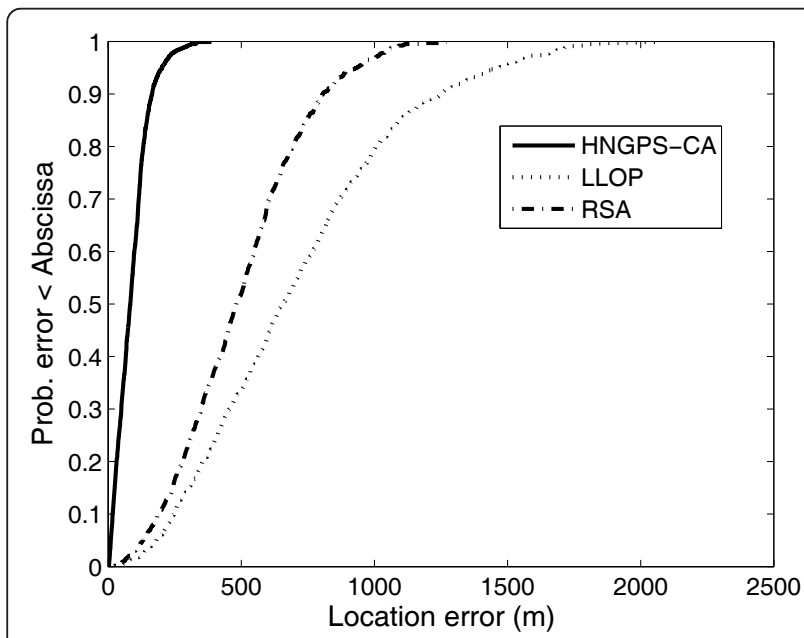

Figure 5 Comparisons of the CDF of the estimation error using the HNGPS-CA, the LLOP and the RSA algorithms in the second case NLOS environment model.

\section{Conclusion}

An algorithm, named HNGPS-CA, is proposed and investigated via simulations for mitigating the effect of NLOS error in outdoor suburban NLOS environment. The HNGPS-CA algorithm locates the MS by minimizing an objective function formed from TOA measurements at three BSs and utilizes the equality constraint provided by the GPS TOA measurement. This proposed objective function of the HNGP-CA algorithm has the guaranteed convexity advantage over the regularly used LS objective function. Simulations results showed that the proposed HNGPS-CA algorithm gave better performance than other positioning algorithms.

\section{Competing interests}

The author declares that they have no competing interests.

Received: 20 November 2011 Accepted: 10 March 2012 Published: 10 March 2012

\section{References}

1. S Venkatesh, R Buehrer, NLOS mitigation using linear programming in ultrawideband location-aware networks. IEEE Trans Veh Technol. 56, 3182-3198 (2007)

2. W Wei, $X$ Jin-Yu, Z Zhong-Liang, A new NLOS error mitigation algorithm in location estimation. IEEE Trans Veh Technol. 54, 2048-2053 (2005). doi:10.1109/TVT.2005.858177

3. $\mathrm{K}$ Yu, Y Guo, Improved positioning algorithms for nonline-of-sight environments. IEEE Trans Veh Technol. 57, 2342-2353 (2008)

4. K-T Feng, C-L Chen, CH Chen, GALE: an enhanced geometry-assisted location estimation algorithm for NLOS environments. IEEE Trans Mobile Comput. 7, 199-213 (2008)

5. S Al-Jazzar, M Ghogho, D McLernon, A joint TOA/AOA constrained minimization method for locating wireless devices in non-line-of-sight environment. IEEE Trans Veh Technol. 58, 468-472 (2009)

6. S Venkatraman, J Caffery Jr, H-R You, A novel TOA location algorithm using LOS range estimation for NLOS environments. IEEE Trans Veh Technol. 53, 1515-1524 (2004). doi:10.1109/TVT.2004.832384

7. D Akopian, J Syrjarinne, A fast positioning method without navigation data decoding for assisted GPS receivers. IEEE Trans Veh Technol. 58, 4640-4645 (2009)

8. G Djuknic, R Richton, Geolocation and assisted GPS. Computer. 34, 123-125 (2001). doi:10.1109/2.901174

9. S Boyd, L Vandenberghe, Convex Optimization, (Cambridge University Press, Cambridge, 2009)

10. P Rooyen, M Lotter, D Wyk, Space-Time Processing for CDMA Mobile Communications, (Kluwer Academic Publishers, Norwell, 2000)

11. J Caffery, A new approach to the geometry of TOA location, in IEEE VTS Fall VTC 2000, (Boston, MA, USA, 2000), pp. 1943-1949

doi:10.1186/1687-1499-2012-100

Cite this article as: Al-Jazzar: Enhancement of wireless positioning in outdoor suburban NLOS environment using hybrid-network-GPS systems. EURASIP Journal on Wireless Communications and Networking 2012 2012:100. 